Boise State University

ScholarWorks

$8-1-2018$

\title{
A Timelapse Camera Dataset and Markov Model of Dust Devil Activity at Eldorado Playa, Nevada, USA
}

\author{
Ralph D. Lorenz \\ Johns Hopkins University Applied Physics Laboratory \\ Brian K. Jackson \\ Boise State University \\ Peter D. Lanagan \\ College of Southern Nevada
}

\section{Publication Information}

Lorenz, Ralph D.; Jackson, Brian K.; and Lanagan, Peter D. (2018). "A Timelapse Camera Dataset and Markov Model of Dust Devil Activity at Eldorado Playa, Nevada, USA". Aeolian Research, 33, 33-43. http://dx.doi.org/10.1016/j.aeolia.2018.04.003 


\title{
A Timelapse Camera Dataset and Markov Model of
}

\section{Dust Devil Activity at Eldorado Playa, Nevada, USA.}

\author{
Ralph D. Lorenz*
}

Johns Hopkins University Applied Physics Laboratory, 11100 Johns Hopkins Road, Laurel, MD 20723, USA.

Brian K. Jackson

Dept. of Physics, Boise State University, 1910 University Drive

Boise, ID 83725-1570, USA.

Peter D. Lanagan

Department of Physical Science, College of Southern Nevada, 700 College Dr., Henderson, NV 89002

*Corresponding Author. Tel. +1 4437782903 Fax. +1 4437788939 Email: Ralph.lorenz@jhuapl.edu

Lorenz, R. D., B. K. Jackson and P. D. Lanagan, 2018. A Timelapse Camera Survey of Dust Devil Activity at Eldorado Playa, Nevada, USA, Aeolian Research, 33, 33-43.

https://doi.org/10.1016/j.aeolia.2018.04.003 


\section{Abstract}

We report a May-June 2015 survey of dust devil activity on a Nevada desert playa using an inexpensive digital timelapse camera. We discuss techniques for exploiting the large volume of data $(\sim 32,700$ images, made publicly-available) generated in these observations, similar to imaging from Mars landers and rovers, noting the diurnal image filesize variations as a useful quick-look metric of weather conditions. We present results from a semi-automated image classification : this classification is available to other workers, for example for benchmarking automated procedures. The acquisition of images at $1 /$ min for some 36 days permits study of the diurnal variation of dust devil activity (e.g. $85 \%$ of the dust devil images [i.e. those images manually classified as showing dust devils] occur between 12:00 and 17:00 ; during the period of peak activity 13:00-15:00 about 7\% of images contain well-defined dust devils of several meters diameter or larger). The data also permit the dependence of dust devil characteristics on ambient conditions. We construct a simple two-state Markov model for the occurrence and persistence of dust devils (a few per cent chance that new dust devil activity appears in the next image; and a $\sim 45 \%$ chance that activity stops) which may help inform strategies for acquiring and interpreting field observations.

Keywords: Dust Devils; Meteorology 
1. Introduction

Dust devils are of interest as a phenomenon in their own right (e.g. Lorenz et al., 2016; Balme and Greeley, 2006) but also as a factor in dust-lifting and air quality more generally (e.g. Gilette and Sinclair, 1990). Dust devil activity has been assessed visually in the past (e.g. Sinclair, 1969 ; Snow and McLelland, 1990; Pathare et al., 2010), with observations recorded by hand in real time. This enterprise is demanding (not merely in time, but in terms of heat and boredom) and yields only crude classifications of dust devil sizes. Photographic surveys, with human operators on-site, can yield quantitative size and trajectory information (e.g. Balme et al., 2012) but are similarly inefficient in terms of their high labor cost.

Modern technology, and specifically digital cameras with large flash memories (Lorenz et al., 2010), allows long-duration visual surveillance of field sites in a manner not dissimilar from that in which visual observations are made from landers and rovers on Mars (e.g. Greeley et al., 2006). Such cameras are a powerful 'force multiplier' in that weeks or months of observations can be made and examined (under office conditions) with only hours or days of effort. Automated image analysis procedures can also be applied to this problem (e.g. Castano et al., 2008), although these are not the focus of the present paper.

2. Methods

2.1 Field Site 
The study site was Eldorado playa (Figure 1), a lake bed conveniently accessible from Las Vegas, Nevada in the Southwestern USA. This location has been the site of numerous prior dust devil investigations (e.g. Pathare et al. 2010; Metzger et al., 2011; Mason et al., 2014; Balme et al., 2012). Previously, we have documented (Lorenz and Lanagan, 2014; Jackson and Lorenz, 2015) statistically robust in-situ barometric surveys of dust devil vortices on Earth at this location, and an evaluation of the populations of dustless and dust-laden vortices using loggers that recorded both sunlight and pressure (Lorenz and Jackson, 2015).

A tradeoff exists in siting a camera to view dust devils. Down-looking views (as from orbit) give precise locations and diameters, but (except via shadows) give little vertical structure information. Horizontallooking cameras expose vertical structure well, but yield no information on the range to the devil and thus image scale : this ambiguity can be removed by stereo imaging (e.g. Balme et al., 2012). A typical elevated vantage point may be, as in this instance, have a generally horizontal view, but there is some distance (range) information to a dust devil from the (foreshortened) vertical coordinate of the base of the dust devil in the image plane. An elevated viewpoint also means that dust devils are seen against a surface background rather than against the sky : in the present location at least, this appears to give typically better contrast. For horizontal viewing in the northern hemisphere, a northward camera boresight avoids looking into the sun, which generally gives poor results. In practice, the practical consideration of choosing a site where a camera can be easily accessed but safely left unattended for long periods may dictate the choice of location. 


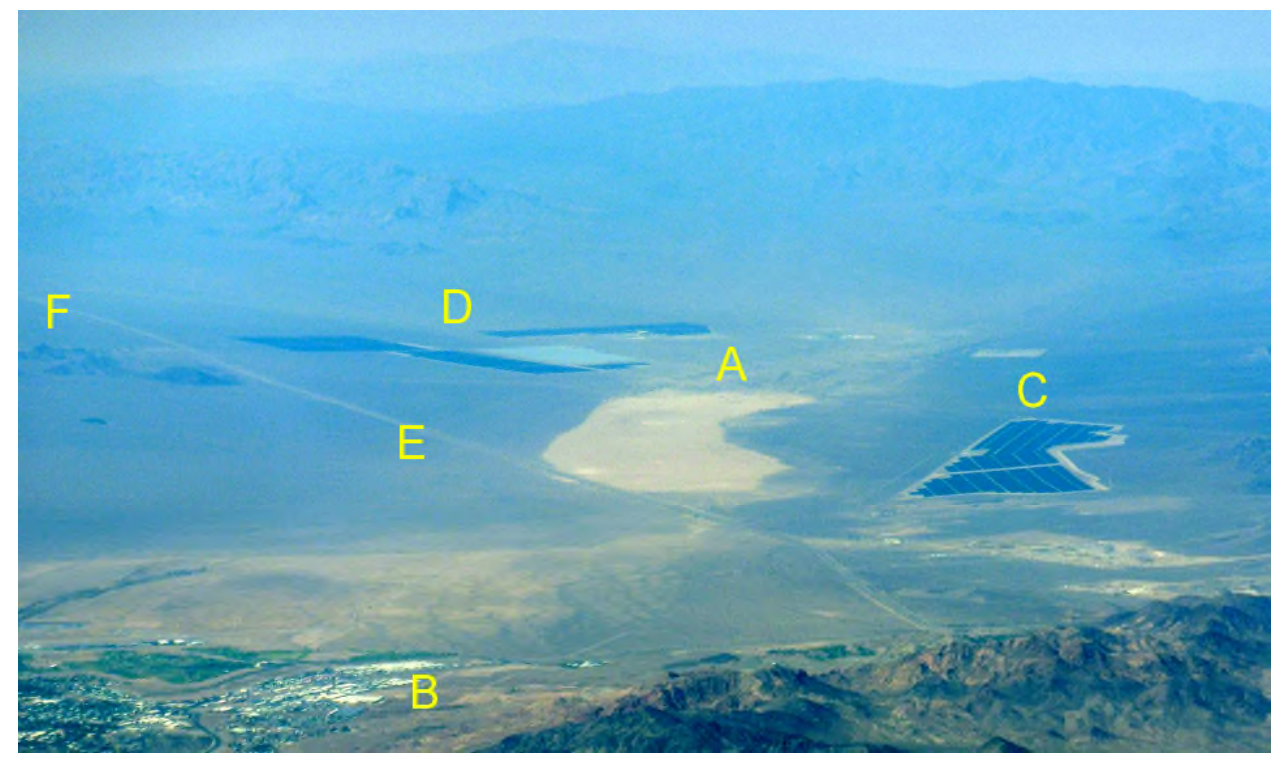

Figure 1. Field site from a commercial airliner looking south-southwest from Lake Mead. Eldoradodry lake $(A)$ is the bright lozenge in center. At the lower left is Boulder City, the CMP04 meteorological station is marked with a ' $\mathrm{B}$ '. The polygonal dark feature $(\mathrm{C})$ to the west of the playa, and the rectangles (D) to the south, are photovoltaic solar power facilities. The faint line running past the left-hand edge of the playa is Route $95(E)$, and the camera location is marked (F). It is evident from this image that winds from the south will have encountered smoother terrain before encountering the playa that winds from the north. 


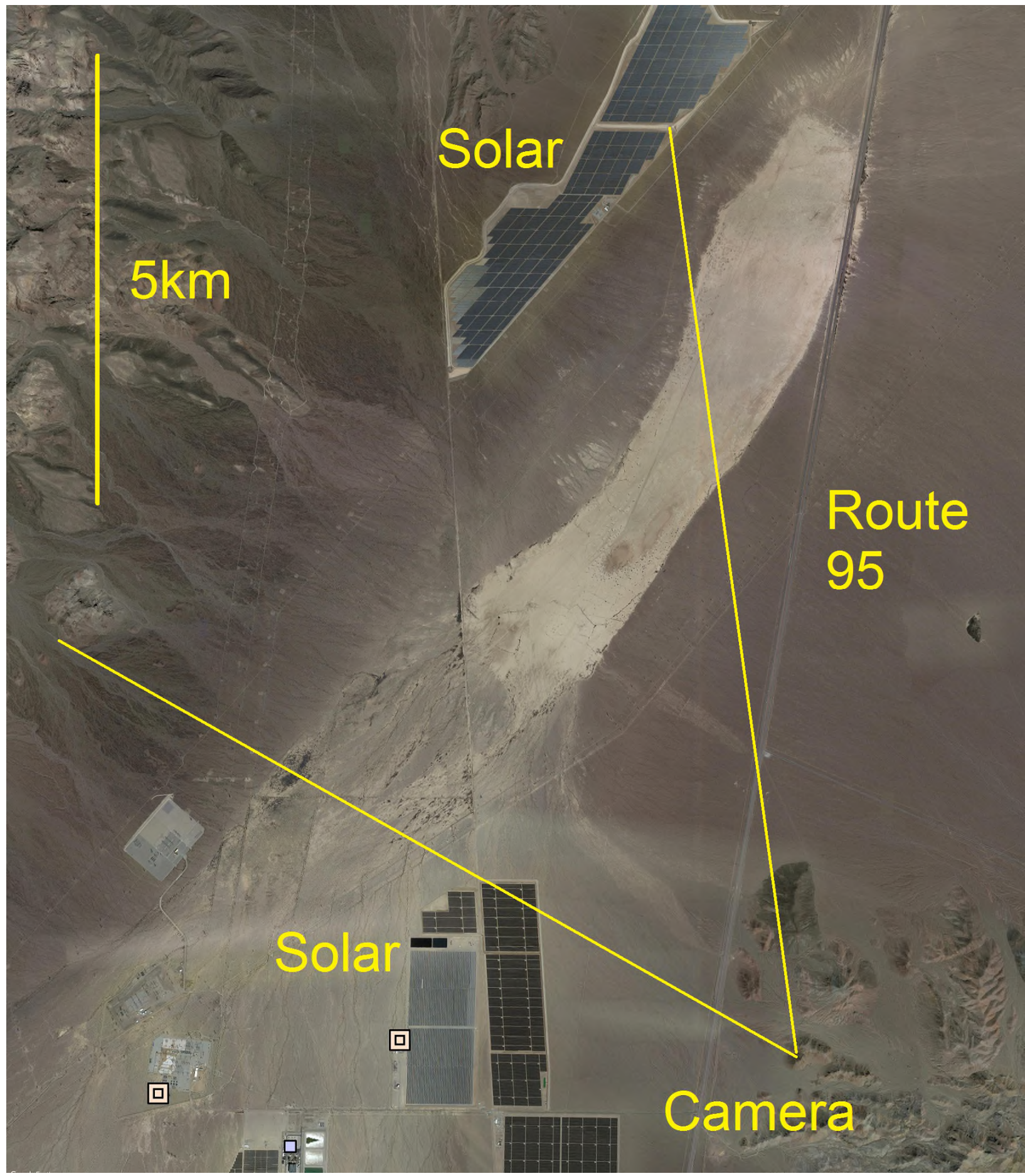

Figure 2. Satellite view, Landsat data courtesy of Google Earth, with the camera location and $45^{\circ}$ field of view shown. The solar power facilities and route 95 serve as useful fiducial marks in the image. 
The camera was deployed in a recessed position among some rocks to protect from direct sunlight and possible human disturbance. The site chosen (and verified with relevant authorities - see acknowledgments), on a small hill to the south of the playa on the other side of Route 95 (Figure 2), has a good view of the playa and is quickly reached from 95 , but requires care to access by climbing on steep slopes with nearby mine workings. Abundant spent cartridges, ceramic skeet fragments, and occasional gunfire noise attest to the informal use of the site for recreational shooting. However, in several years of experimental deployments, hardware attrition has been minimal, and no adverse effects on personnel (beyond occasional scrapes on the rocky slopes) have been encountered.

\subsection{Camera Instrumentation}

We have previously noted (Lorenz et al., 2010) the application of then-emerging digital timelapse camera technology exploiting flash memory to perform surveys of transient meteorological phenomena such as dust devils or playa flooding. Since that work, with somewhat primitive custom-built 
equipment, new consumer camera products have provided progressively higher-quality images and larger memory capacity, with more image acquisition flexibility (e.g. video bursts) and better packaging.

For the present study, we used a Cuddeback 'Attack' camera, a unit with which we have had satisfactory experience in meteorological monitoring (e.g. playa flooding and rock movement at Death Valley's Racetrack Playa - Lorenz et al., 2014). Although principally sold as a trail camera for monitoring wildlife (its motion detection and night-time near-IR illumination capabilities were not relevant in our application), its robust waterproof housing, accommodation of D-cells for long battery life and ease of set-up in the field have generally given good results : an example image is shown in Figure 3.

As discussed in Lorenz et al. (2010) there are inevitable tradeoffs in any timelapse program. In particular, the image cadence and survey duration are usually limited by memory size and/or battery capacity, with image resolution sometimes an adjustable parameter. For playa flooding, an hourly or half-hourly cadence is adequate, but for faster transient events such as aeolian ripple migration (e.g. Lorenz and Valdez, 2011), a timescale of a few tens of seconds to a couple of minutes is more appropriate.

We have previously trialled 5-minute surveys at this site. While this approach is reasonable for an overall activity census (and allows multi-month unattended operation, since the memory card fills and the batteries run down slowly), this interval is too long to reliably track individual devils: Following Lorenz (2013), the typical longevity of a dust devil varies as $t=40 d^{0.66}$, with $t$ the duration in seconds and $\mathrm{d}$ the diameter in meters; thus only relatively rare devils $20 \mathrm{~m}$ diameter or larger will appear in multiple frames collected at 5-minute cadence.

Hence, in order to follow the life-cycle of individual devils but still retain a long enough observation period to be sure of catching a variety of conditions, the current survey was conducted with a 1-minute image cadence (thus typical $>5 \mathrm{~m}$ devils should appear in more than one frame). At a distance of $\sim 5 \mathrm{~km}$, 
a $5 \mathrm{~m}$ devil subtends 1 milliradian (and thus should subtend about 3 pixels, see below.)Of course, a higher-resolution camera could in principle detect smaller, more abundant dust devils. Lorenz (2009) shows that the total number of devils varies roughly as the inverse-square of the size threshold, so a camera able to resolve $2 \mathrm{~m}$ devils might detect (25/4) 6 times more devils. However, it would require either a detector with 6 times as many pixels (with corresponding increase in the demands on the memory capacity of the system, and on the operator performing image classification), or would have to be looking at a smaller area.

The camera was left unattended over the summer period and retrieved some months later. Although the memory card could have accommodated more images and the batteries were not depleted, 32767 images (including some spurious images acquired prior to and after deployment) were recorded, possibly limited by a filesystem constraint (SD memory cards may be formatted with different filesystems, e.g. FAT32, and care must be taken to select the appropriate one per manufacturer's instructions).

The set of $\sim 32,700$ useful images (13.5 Gbyte of data) were acquired in $\sim 36$ days of operation. Since images were only recorded by the camera when light was above a certain level, there were about 1000 images per day, roughly between $5 \mathrm{am}$ and $7 \mathrm{pm}$. Image quality (and thus file size) is a controllable parameter - for the present survey, images were $2592 \times 2000$ pixels (i.e. $~ 5$ megapixels) with a typical filesize of 454 kiloBytes (i.e. the three-color information is encoded at $\sim 1$ bit/pixel ). Typical daytime exposure time was $1 / 1000$ s at $\mathbf{f 2 . 7}$. The image data are available for download at http://lib.jhuapl.edu 
An example image is shown in Figure 3. In this frame (25844) a prominent dust devil is visible, one of the largest well-formed dust devils in the image sequence. Measurement with the profile tool in ImageJ shows that the devil is about 70 pixels wide at its base - and thus is about $100 \mathrm{~m}$ in diameter. The contrast in the red channel of the image is about $30 \%$ (about $230 \mathrm{DN}$ against a background of $180 \mathrm{DN}$.)

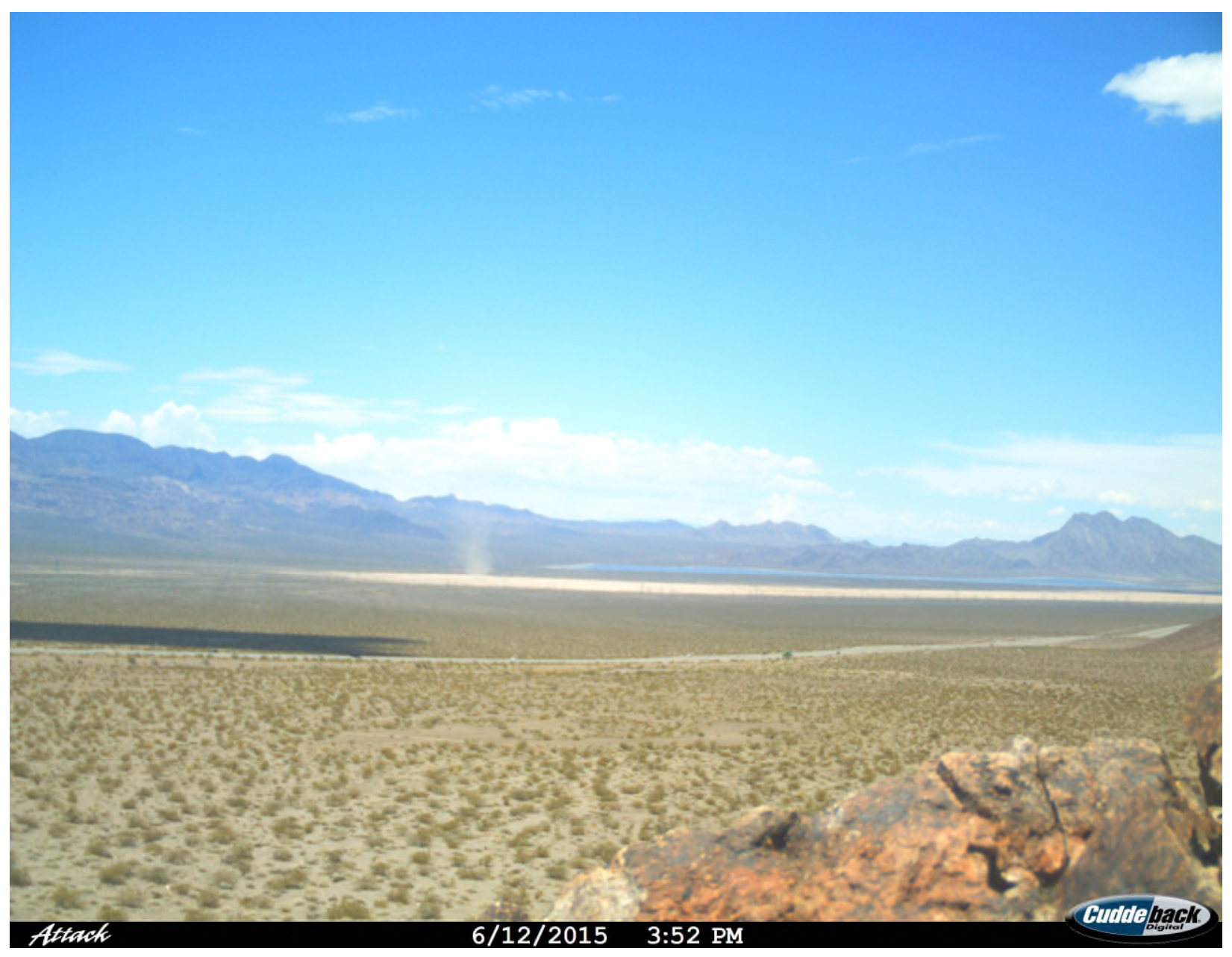

Figure 3. Image 25844, in early afternoon (note that the timestamp at the bottom of the image is Eastern time, 3 hours ahead of local) showing route 90 in the foreground and blue skies. The playa, and the solar power installation beyond, are visible as near-horizontal features in the center of the image; a cloud shadow is at center left. A large ( $100 \mathrm{~m}$ diameter) dust devil is visible a third of the way across the image - note that its visibility is due to the high contrast between the bright dust and the darker hills beyond; the dust is less obvious against the cloudy sky or against the playa itself. The good definition of the dust devil in this image earned it a categorization as 'category 4' (see later). 


\subsection{Analysis Methods}

To examine every frame individually 'by hand' would be very onerous (albeit less time-consuming than observing in the field!), but the confidence of automatic detection algorithms is uncertain. Thus a 'reconnaissance' semiautomated approach was adopted, wherein a computer script (implemented in the Interactive Data Language IDL) displayed images sequentially for about a half second at a time, without waiting for user input. The operator could flag an image as of interest by using the computer mouse. Although demanding close attention of the operator, this approach allowed the entire dataset to be processed in 2-3 hours. It was noticed that there was a slight response delay, in that the sequence advanced by $2-3$ frames by the time the operator registered activity by mouse action, and by 1-2 frames after the activity ceased - this is easily corrected, however (see below).

This pre-screening identified about 1800 frames out of the 32,700 (i.e. about $5 \%$ of the frames were identified as having some dust activity). Some of these were false alarms (e.g. dust raised by vehicles, or rain). Some small, faint and/or brief dust devils were not flagged.

A second computer script then presented each of the flagged images (and in addition, two images prior and one after each flagged image, to take into account the response time issue above), and displayed it until the operator designated it by a keyboard press in one of 3 principal categories (see table 1 chosen since three fingers can rest continuously on the keys for easy input - a fourth category was only occasionally used). 


\begin{tabular}{|c|l|}
\hline Image Category & Description \\
\hline 0 & $\begin{array}{l}\text { Not flagged for further scrutiny (designation only } \\
\text { specific to this analysis implemenation) }\end{array}$ \\
\hline 1 & $\begin{array}{l}\text { Examined and judged not to be of interest - dust } \\
\text { features not present, small or obviously artificial }\end{array}$ \\
\hline 2 & $\begin{array}{l}\text { Dust features present. May be unstructured dust } \\
\text { devils, or dust 'blows' related to rainstorm or other } \\
\text { winds, plume from prior dust devil no longer } \\
\text { lifting. Possible occasional artifacts (e.g. vehicle } \\
\text { plume) }\end{array}$ \\
\hline 3 & $\begin{array}{l}\text { Well-defined dust devil(s) present ;morphology } \\
\text { and/or size can be determined }\end{array}$ \\
\hline 4 & $\begin{array}{l}\text { As (3) but image flagged as especially visually } \\
\text { appealing }\end{array}$ \\
\hline
\end{tabular}

Table 1. Image classification categories used in our semi-automated procedure.

It should be noted that these classifications were generally applied on the appearance of each individual frame. The presence of a dust devil is easier to infer in a given image in a sequence, in that the eye naturally tracks moving features. No attempt was made to correct for this effect, however.

The playa area observed is roughly $6 \mathrm{~km}^{2}$ in area : an empirical fit to a wide range of terrestrial and Martian visual surveys (Lorenz, 2009; 2013) suggests that the observed number density of dust devils ( $\mathrm{N}$, in devils per $\mathrm{km}^{2}$ per day) relates inversely to the area $\left(A\right.$ in $\mathrm{km}^{2}$ ) under study ( $N$ 50/A). This follows from typical detections requiring a certain angular width threshold, and from the power-law size distribution of dust devil diameters. On this basis we would expect to observe about 50 dust devils per day. Of course, many small dust devils will be present but not detected. 


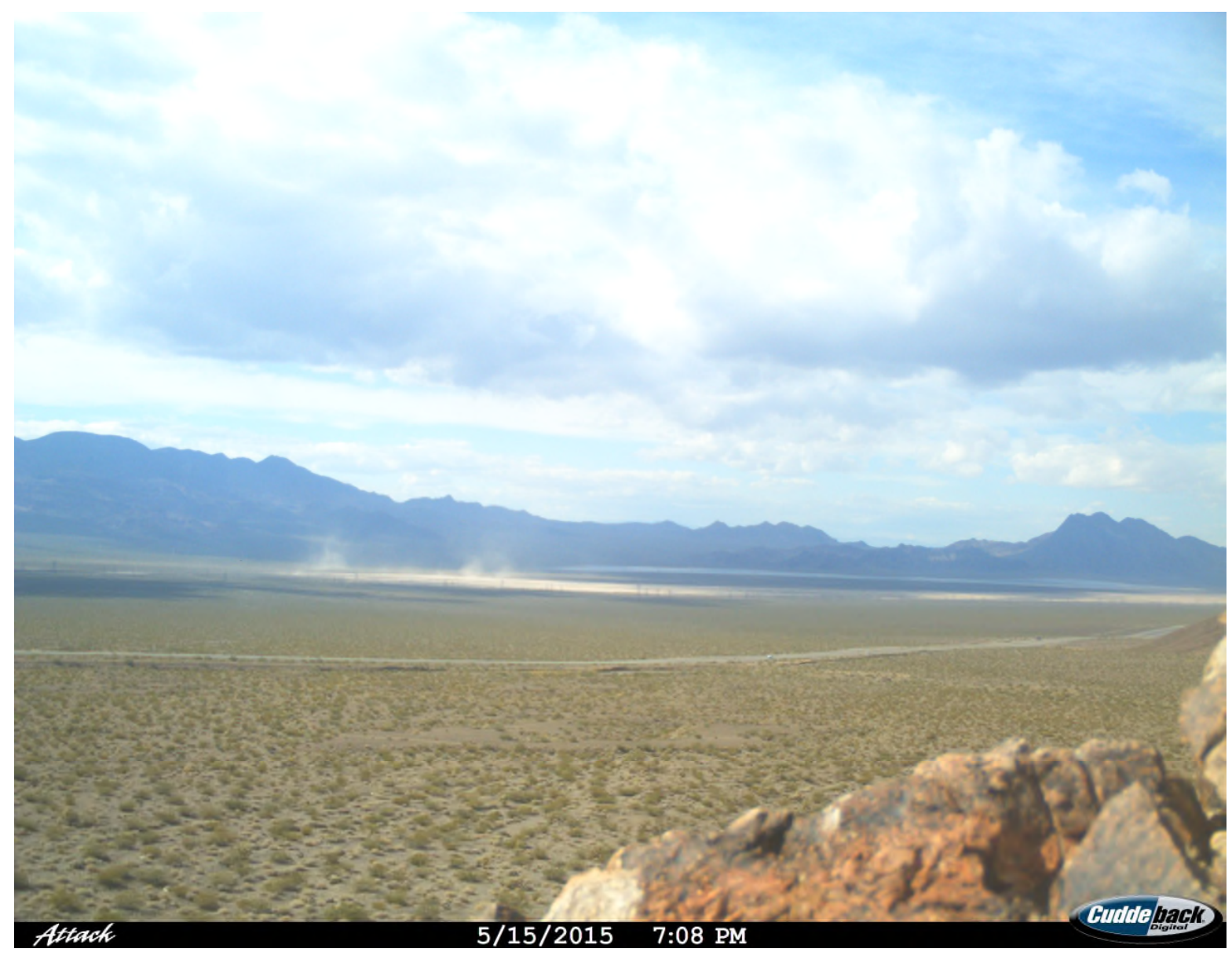

Figure 4. An example Category 2 image (01023) showing poorly-structured dust raising - examination of the image sequence shows dust and new dust sources moving left-to-right, at the same speed as the clouds above. It seems that updrafts associated with the clouds can trigger dust-raising. While occasional transient dust devils arise in these conditions, they rapidly disappear : either vortices are weaker due to reduced surface heating, or the strong winds are shearing them out.

\subsection{Weather Conditions}


Meteorological data at the CMP (Community Meteorological Project, operated by the Department of Energy) weather station in Boulder City (see Figure 1) was downloaded for the period observed by the timelapse camera. Data are acquired every 15 minutes and are summarized in Figure 5. The station is only about $16 \mathrm{~km}$ from the playa, and the terrain between is relatively unobstructed, so the record is useful as a general indication of weather conditions. This is especially true when wind is coming from the south, as is usually the case. Note, however, that for typical wind speeds, an air parcel crosses the weather station about half to one hour after passing the playa. 

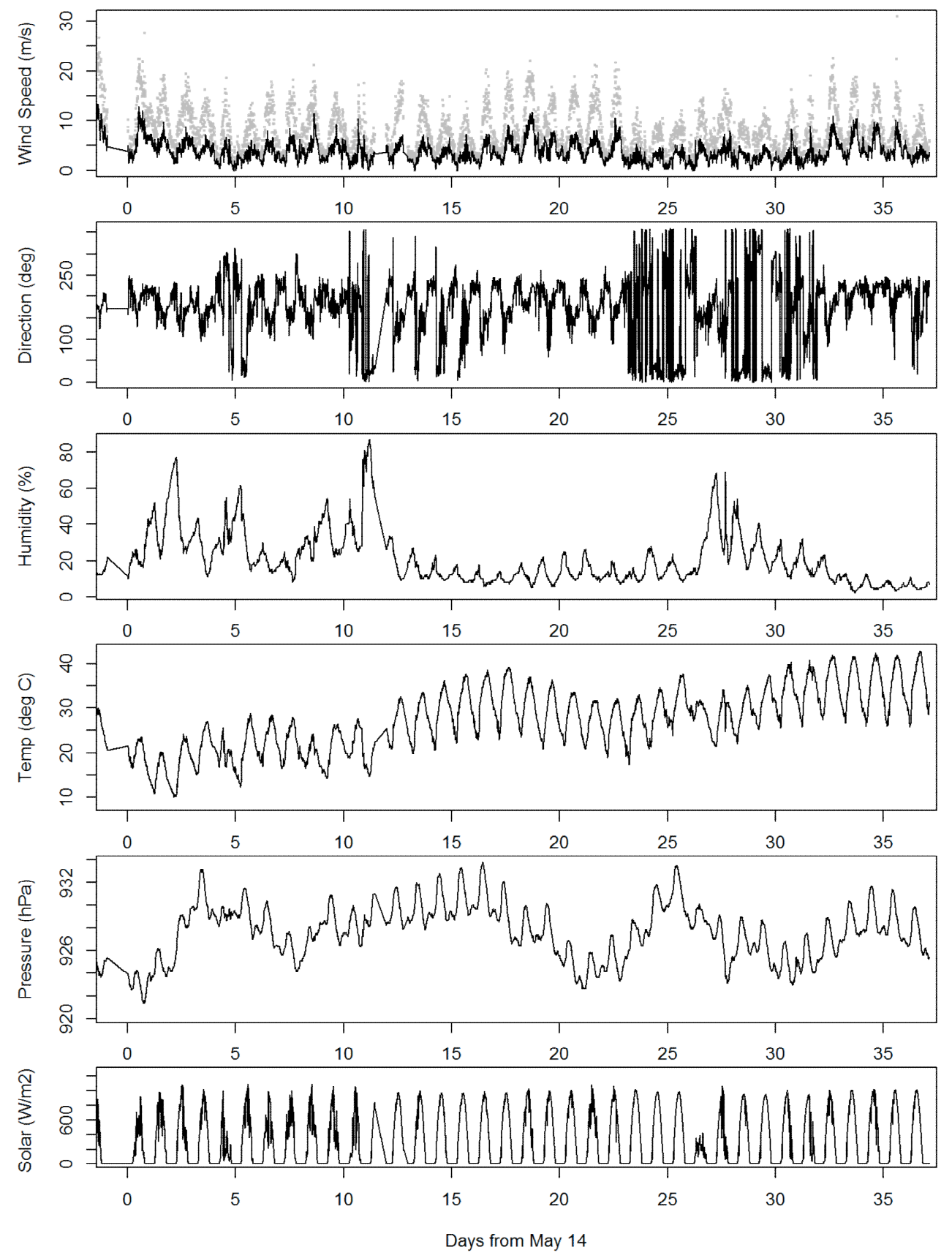

Figure 5. Weather conditions for the study period, as recorded by the CMP Boulder City meteorological station at 15 minute intervals. Wind speed (top panel: grey=gust, black=mean) shows a remarkably invariant pattern. The first 11 days have cooler and more disturbed conditions, with variable clouds indicated in the solar flux (bottom) and higher humidity. Days 13-25 show fine conditions with clear skies, low humidity, and southerly winds. There then follow a few days of disturbed (moist) conditions, and then days 33-36 resume the 'regular' pattern. 


\section{Image Analysis Results}

We recognize other authors may devise their own human or automated analyses of the image data, and it is our goal in making the images available that such analyses be made. It is possible, for example, to track the evolution of individual devils as they migrate across the playa, grow and decay (see Figure 6 ). It is evident in this sequence that the dust devil grows in both diameter and height at first, then tightens (i.e. reduces its diameter while maintaining a large height). It may be interesting to explore whether this same behaviour is exhibited by Large Eddy Simulations (LES) of dust devil vortices. It is also interesting to observe dust devils migrating simultaneously in opposite directions - in low ambient winds, the pseudorandom local winds of the boundary layer convection pattern may dominate: it is these winds (see also Lorenz, 2013) that can cause the cycloidal migration pattern of some dust devils, evident in their tracks on Mars (e.g. Reiss et al., 2016). 

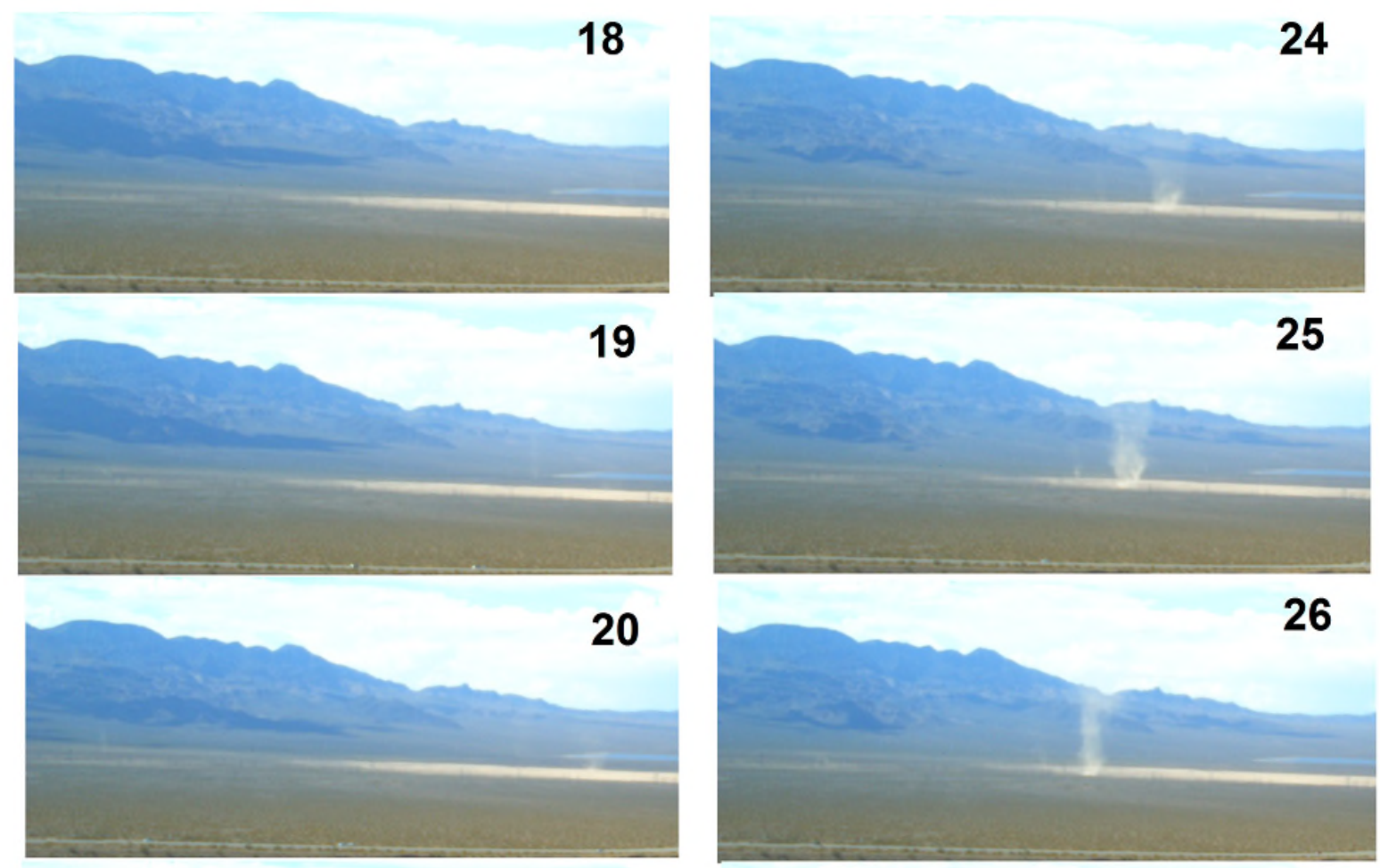

26
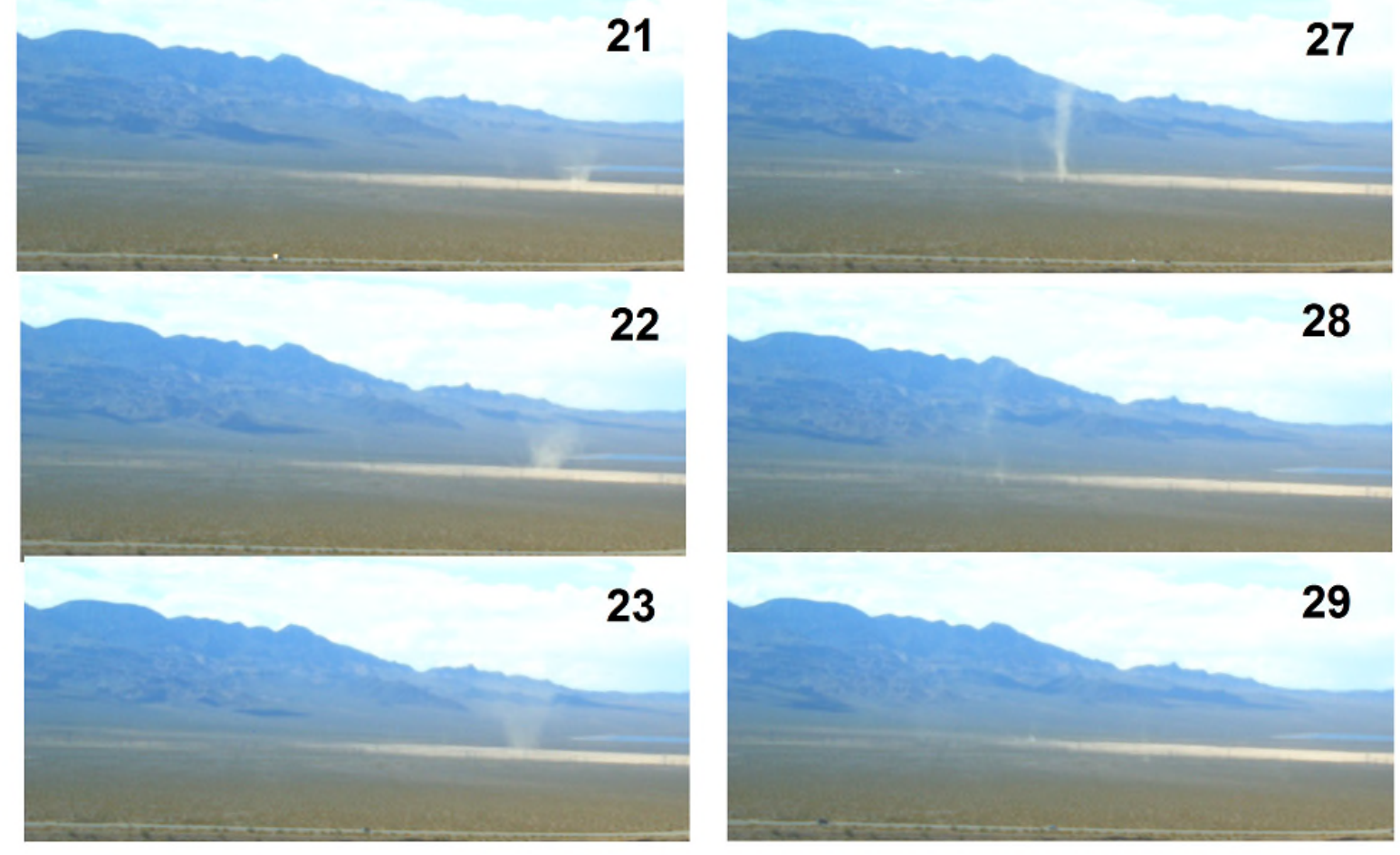

Figure 6. 12-minute sequence showing the development and migration of a dust devil. Devil forms in 
frame 20 and broadens into a conical form, becoming fainter in frame 23. Devil picks up more dust and maintains a more cylindrical form through frame 26 , becoming taller and narrower while migrating to the left (south). Devil disappears (fades, rather than being advected out of view) by frame 29. Images are subframed $(1400 \times 600$ pixels, upper left is coordinate $(1,800)$ of original images 26018 to 26029 . A second small devil can be discerned in frames 25-27.

The classification process described in section 2.3 yields an image list (available at http://lib.jhuapl.edu). With these classification data, which are the subject of the remainder of the paper, we are able to address several questions.

First, the dataset contains 2368 images flagged as showing dust activity - in other words, about $7 \%$ of the time (this includes early morning and other periods when dust would not be expected). If one limits the time to definite dust devils (category 3 or 4 ) then the result is 698 images, or $2 \%$. Figure 7 shows a summary of activity in category 2 or higher - typically there are around 100 images (category 2 or above) per day, but a few days towards the end of the period show three times as many images showing dust activity. The figure also indicates that on days when dust devils occur, there are typically 10-50 images showing them. We have not counted individual dust devils in images, but considering that typically one or two devils may be present, and assuming that typically a dust devil lasts one or two minutes in the field of view (see Figure 11 later), then this suggests between 5 and 100 dust devils per day. This is broadly consistent with the expectation from previous work which suggests around 50 devils per day in any survey.

The diurnal variation is shown in Figure 8 - peak activity is between 13:00 and 15:00 hrs. $90 \%$ of the category 3 or 4 images are acquired between 11:00 and 17:00, suggesting field surveys conducted over that period will be $\sim 90 \%$ efficient (compared with the present survey), although the efficiency degrades only slightly, to $~ 85 \%$ if a survey does not begin until $12: 00$. 

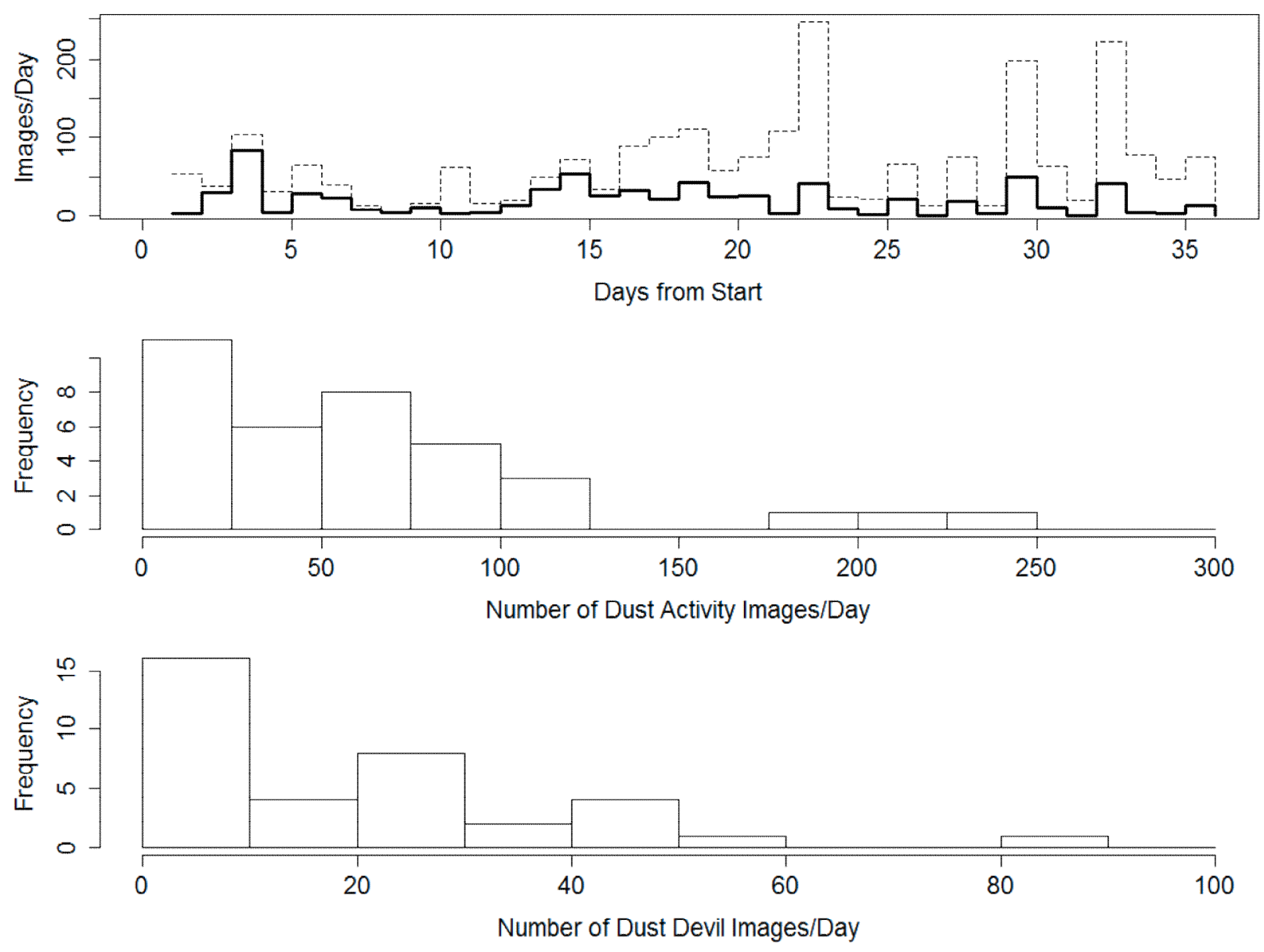

Figure 7. The number of dusty (category 2 , dashed line) and dust-devil (category $3 \& 4$, bold line) images per day during the survey - activity was most consistently in the middle of the observing period (upper panel). Generally, as also shown by the histogram in the middle panel, there are 50-150 dusty images, although three days are exceptional. Similarly, the lower panel shows that typically 10-50 dust devil images (category 3) occur per day, except on days when there are none or almost none. One day was exceptional 


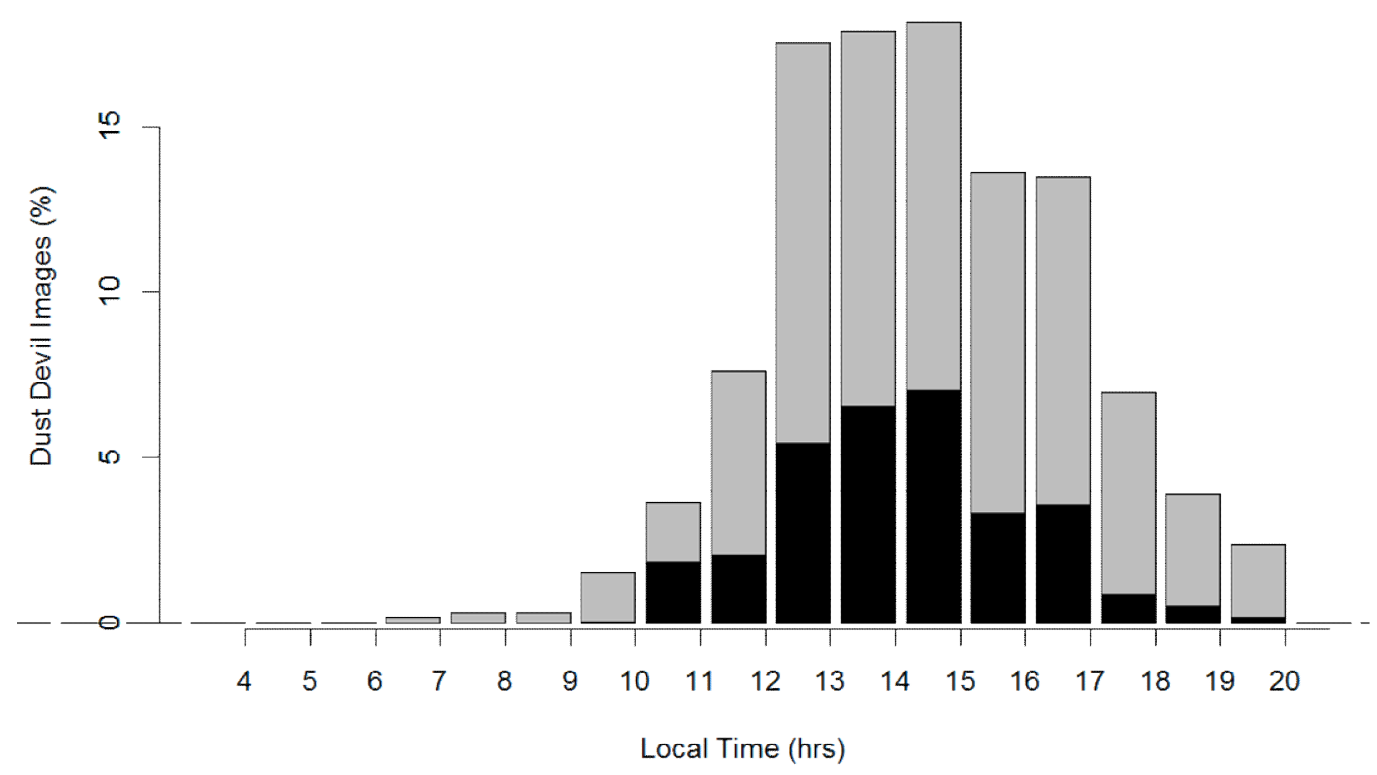

Figure 8. The fraction of images taken during a given hour that show dust devils in category 2 or above (grey) or category 3 (black). In the early afternoon, a given image has an almost $20 \%$ chance of including some dust activity, or about a $7 \%$ chance of showing definite dust devils.

A next question centers around the dependence of dust devil activity on ambient conditions (e.g. Sinclair, 1969; Rafkin et al., 2016). It is qualitatively easy to understand that clear conditions favor strong surface heating and thus the boundary layer convection of which dust devils are a part. It is also obvious that dry conditions favor dust lifting. Figure 9 shows the occurrence of dusty and dust-devil conditions in the image sequence, together with the humidity and wind direction (the most variable quantities in the previous plot). The period of consistent dust devil activity in the middle of the survey (roughly days 10-25) is associated with low humidity conditions, with winds from the south. Dust devil activity is suppressed on days of high humidity and/or wind variability.

One might study ambient weather conditions as indicated in the images themselves (e.g. one could examine the sky color by looking at the red, green and blue intensity values of say, the upper left corner 
of each image). This requires, however, the loading of each image. A quick reconnaissance of a large dataset from a timelapse camera such as that used in this survey can be achieved, however, simply by looking at the filesize of each image, which is available without loading the image itself. This is because the camera performs JPEG (Joint Photographic Experts Group) compression on the image - the image is converted into frequency space by a Fourier transform and a subset of these Fourier coefficients are stored; the number of coefficients selected is a measure of the image quality. The file storage process performs a Huffman coding step (i.e. lossless compression) of the Fourier coefficients, and this coding scheme requires more data if there is high entropy in the image (i.e. lots of varying detail), giving large file sizes. On the other hand, images with wide areas of bland color have low information content (low entropy) and therefore small file sizes. Thus, the file size is a metric of image content, with the obvious corollary that similar images will have similar filesizes. One clear day should look much like another, and thus the filesizes should be similar. The image filesize varies over the course of the day, due to varying brightness, the presence of shadows etc., but the pattern of filesize change over the course of a clear day will be constant. It can be seen in Figure 9 that a repeating pattern of filesize variation occurs in the middle of the survey period, when there was a series of clear days, while the cloudy/stormy condiitons at some other times are also revealed by irregular filesize changes. Whether the filesize changes on clear days are sufficiently reproducible to allow detection of dust devils is left for future work, however. 

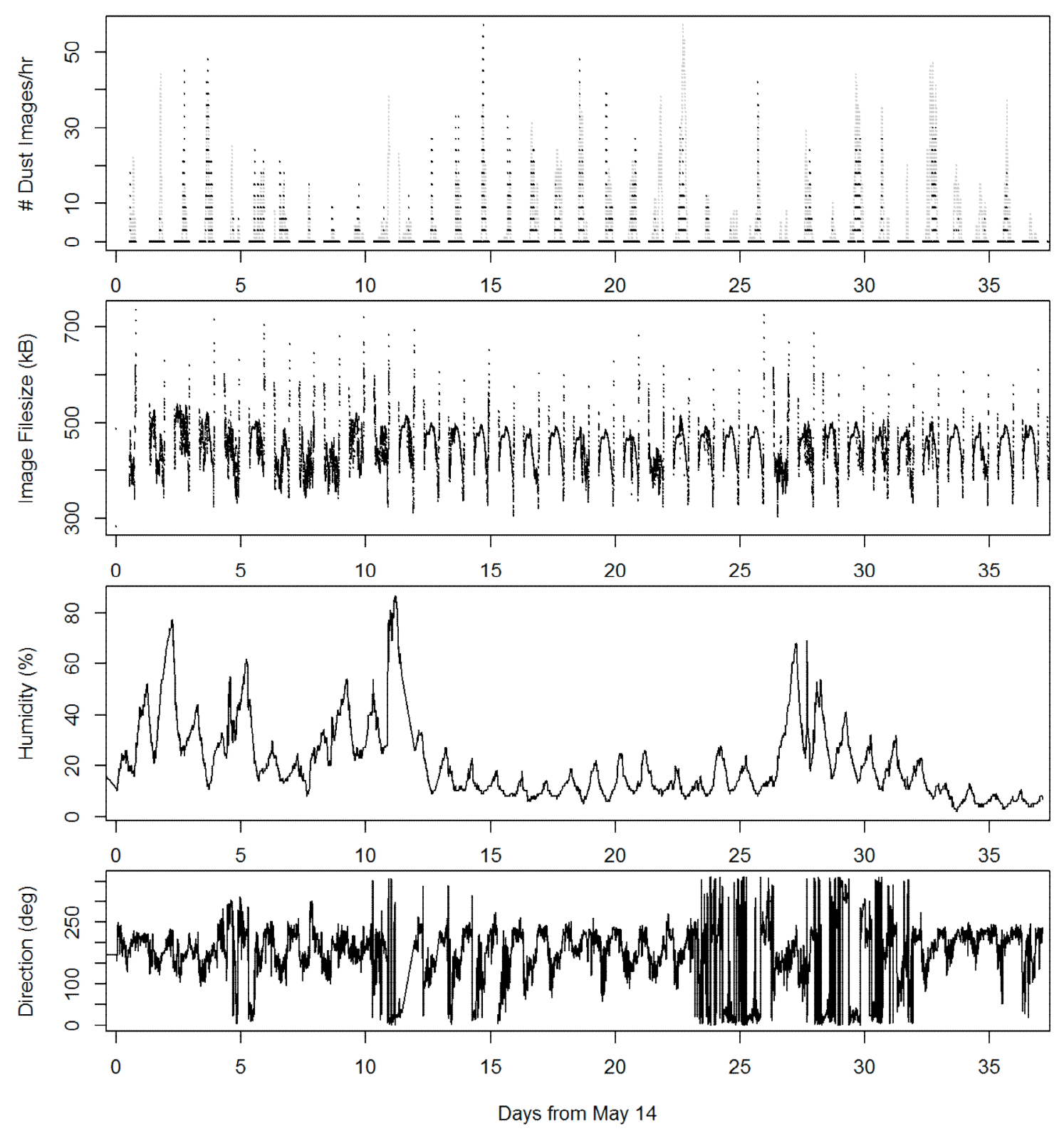

Figure 9. Dependence of dust activity on weather. The top panel shows a 60-min running average of the number of images in categories 3/4 (dust devil, black points) or 2 (dusty, grey). The second panel shows the image file size - there is an evident diurnal pattern to the filesize as the image content varies with illumination : this pattern is most reproducible in the day 12-26 period when conditions were clear. Cloudy conditions e.g. the high-humidity period in days 5-10, have a more variable filesize pattern. It can be seen that the central period of high dust devil activity is associated with low-humidity conditions and winds generally from the south. 
4. Dust Devil Persistence and a Markov Model

Returning to the manual image classification results, Figure 10 shows a close-up of late afternoon sequences of image class, in the raw record and as a running average. It can be noticed that the dustdevil events are not randomly distributed throughout the observing sequence, but rather occur in clusters. This makes intuitive sense, sometimes it is favorable for dust devils to form, and sometimes it is not. 

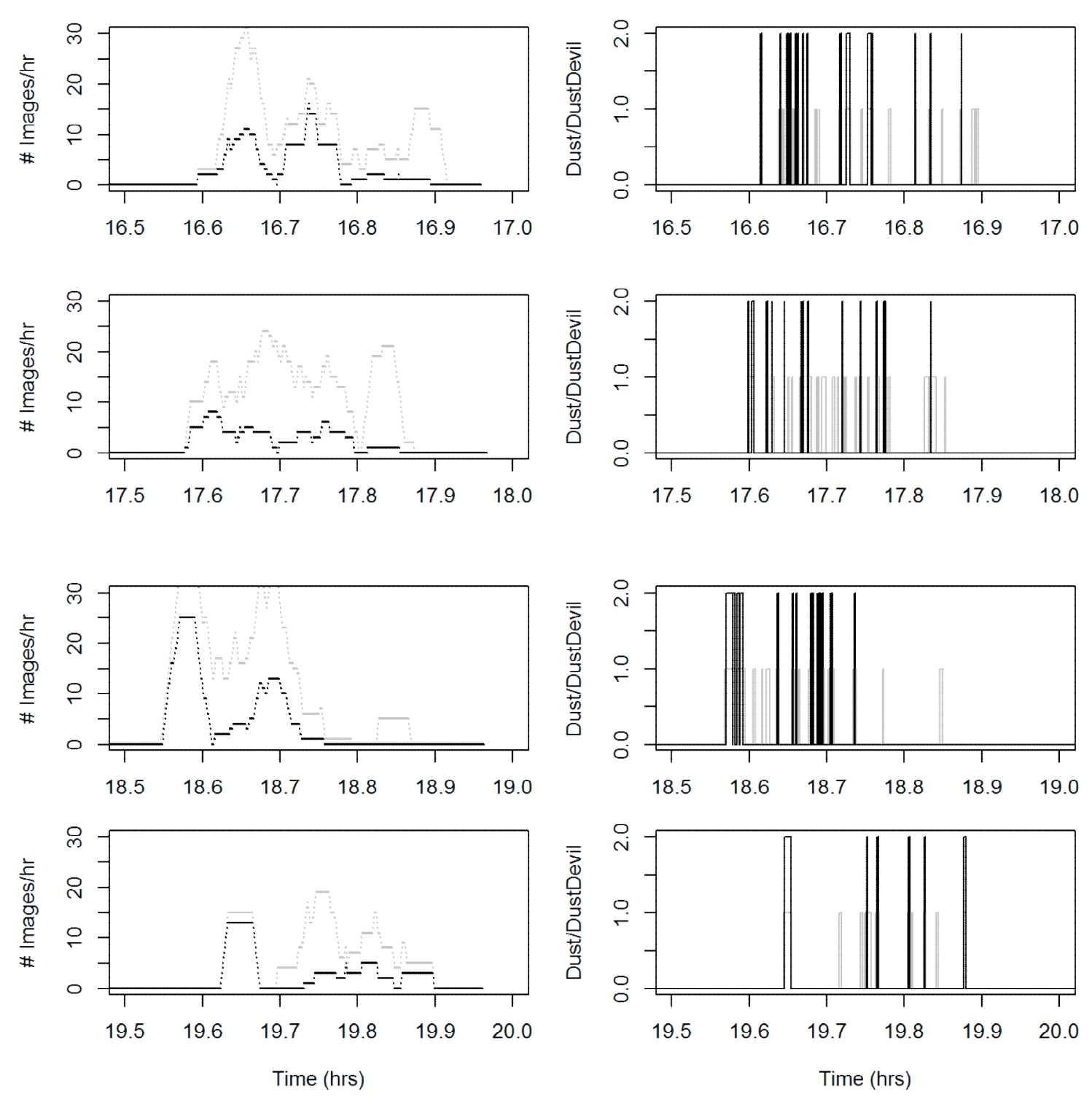

Figure 10. Dusty (grey) and dust-devil (black) images on four of the high-activity days (15-19). Left panel is the running-average of the number images in a one-hour period classified as dusty ( 2 or higher, grey) or dust-devil (black). The individual classification sequence is shown at right - a black bar to 2 denotes a category 3 or 4 image (dust devil), a grey one to 1 denotes a category 2 image (dust) - it is apparent that dust devil images are not randomly scattered through the observation period, but are clustered in 'bursts'. 


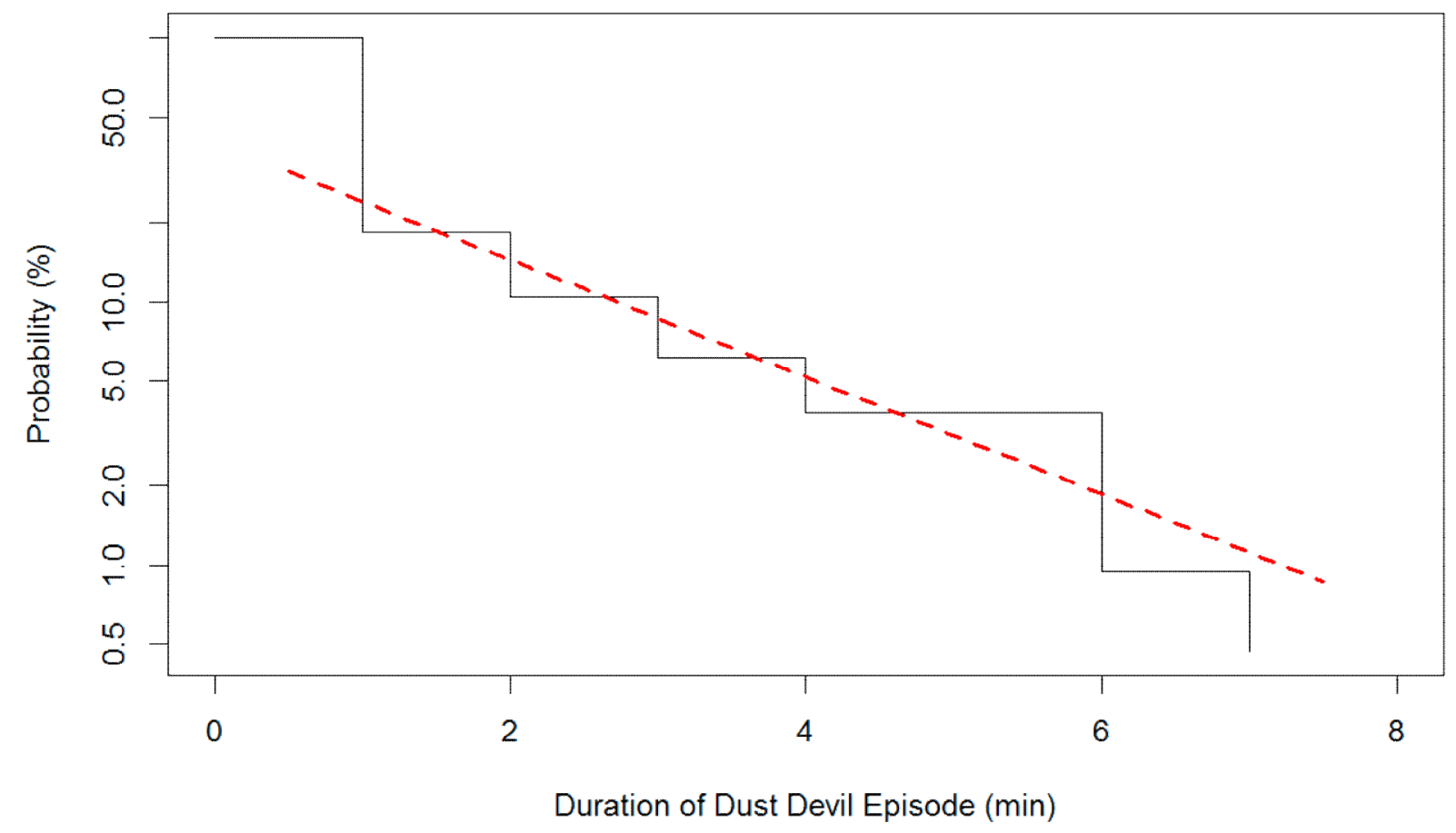

Figure 11. The distribution of run lengths of image sequences of category 3 or higher (i.e. definite dust devils). The red line is an analytic function (see text) with each additional minute causing the probability of encountering such a sequence to decrease by a multiple of $55 \%$.

The category sequence was interrogated with the function 'rle' (run length encoding) in the data analysis package ' $R$ ' ( $w w w . R$-project.org) to determine the distributions of persistence of dust devil conditions by picking out contiguous strings of values. This showed that the image sequence contained $\left(\begin{array}{llll}212 & 39 & 13\end{array}\right.$

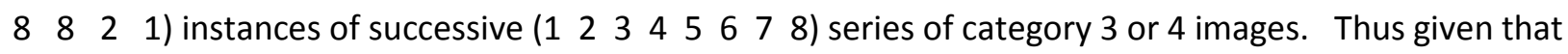
a dust devil has been observed in one image, there is a $(39 / 212)=20 \%$ chance that it or another dust 
devil will be seen in the next image. Figure 11 shows the histogram of these values, and a power law fit by eye, namely $P(x)=p^{x}$, where $x$ is the duration in minutes and $p^{\sim 0.55}$. Note that the presence of a single image of dust devil conditions allows the rather large range of durations of 0 to 1.99 minutes, and thus fitting the first datapoint is less important than fitting the slope. The value of $p$ can then be taken as the state transition probability from < dust devil $>$ to $<$ no dust devil $>$ in a Markov chain (see Figure 12). This transition can be effected either by the decay of a dust devil in-situ (it fizzles out in vortex intensity, or it moves to an area with no available dust so becomes invisible), or by the advection of the dust devil out of the field of view. One or other of these processes may dominate depending on the ambient windspeed, with advection loss being more significant in stronger winds. Of course, two successive $<$ dust devil > images would be obtained if a dust devil in the first were advected out of the field of view, but replaced by another that was advected in from the other side. 


\section{El Dorado Dust Devil Markov Model (1-minute cadence)}

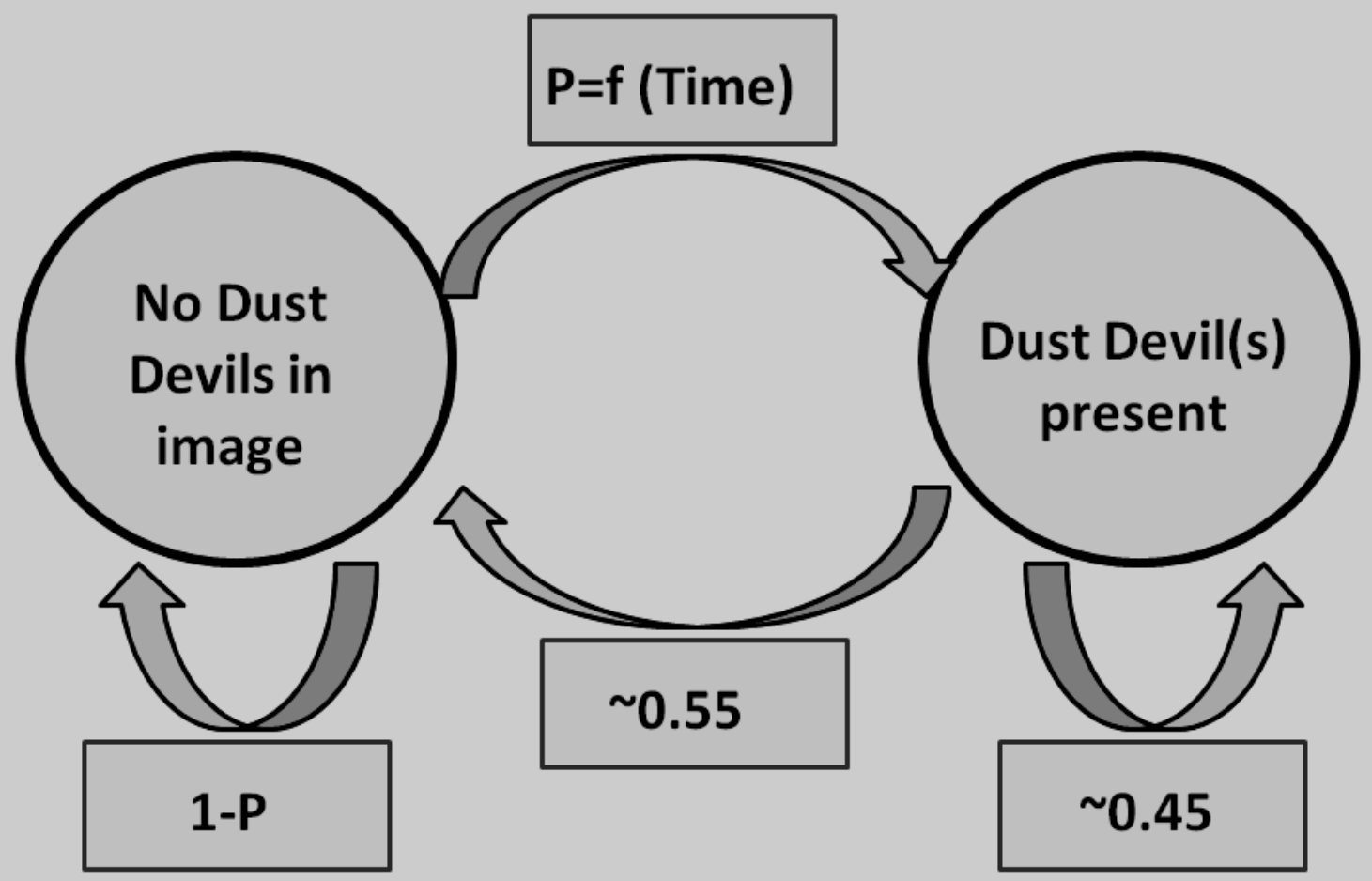

Figure 12. Two-state Markov model. As discussed in the text, the probability for persistence of the dust devil state is $\sim 45 \%$ for each minute image interval. The appearance of the dust devil state has an overall probability in the dataset of a few per cent, but is strongly dependent on time of day.

The state transition model can be completed by calculating the probability of transitioning from $a<$ no dust devil> state to a <dust devil> one. A quick estimate (since this probability is low overall) is simply to divide the total number of <dust devil> sequences (307) by the total number of images (32700), yielding $\sim 0.9 \%$. However, this assumes uniform occurrence throughout the image sequence (i.e. 0400 to 2000 hrs). More useful is to use the time-of-day dependence indicated in Figure 7 : the black (dust devil) 
time-of-day population is fit readily with a Gaussian with a mean of 14.0 and a deviation of $1.82 \mathrm{hrs}$. In other words, most dust devils are imaged within about 2 hours of $2 \mathrm{pm}$ local time. Since each sequence is only a few minutes long, the distribution of times of day of the transitions (start of dust devil sequences) is indistinguishable from the distribution of the images with dust devils. The distribution peaks (Figure 7) at about 7\%; thus the peak state transition probability $P_{\max }$ for the whole dataset is $7 / 0.43=16 \%$ (the factor 0.43 arises from the number of image sequences [307] to total images [698] : 307/698=0.43). Thus the state transition probability may be written $P(t)=0.16 * N((t-14), 1.8)$ where $N(x, s)$ denotes the Gaussian distribution with mean $\mathrm{x}$ and standard deviation $\mathrm{s}$, and $\mathrm{t}$ is the local time in hours. On the other hand, since this period includes several dust-devil-poor days, the function for days on which conditions are known to be favourable may be increased, perhaps as high as $P_{\max } \sim 25 \%$.

Four instantiations of the model (which can be implemented in only 9 lines of ' $R$ ' code - see Appendix) are shown in Figure 13 : it is seen that the model output closely resembles the field observations. 

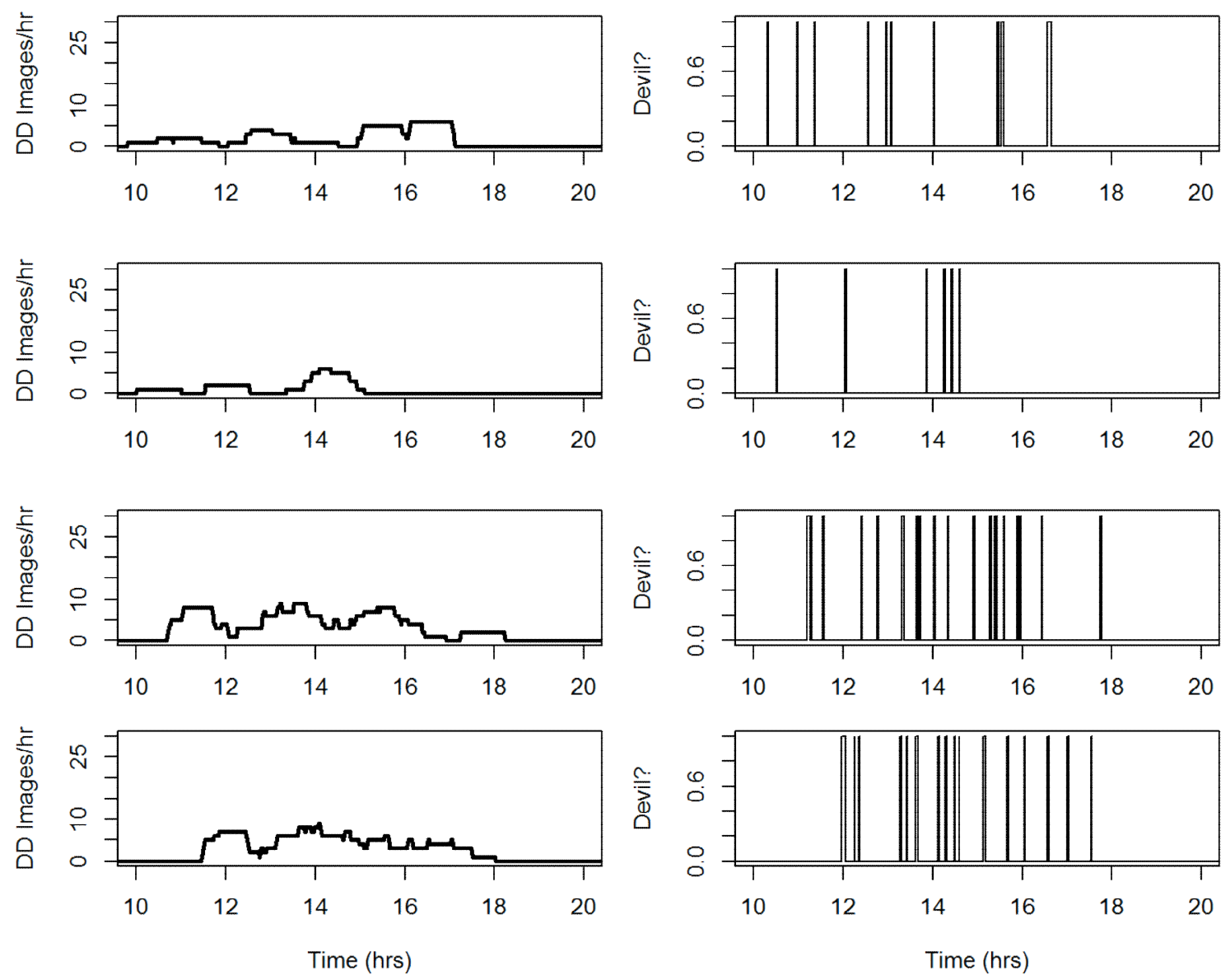

Figure 13. Four instantiations of the Markov model, with dust devil frames shown at right and images/hr at left : this portrayal is directly comparable with Figure 8. The top two instantiations have $P_{\max }=0.15$, and the lower two with $P_{\max }=0.3$. It is seen that the model output appears to represent the data very well.

\section{Conclusions}

A dataset has been acquired and made available which exposes the evolution and statistical properties of dust and dust devils at the Eldorado site. A manual classification of images has been made, which can 
be used as a benchmark for automated image analysis procedures. The image filesize variation is a useful 'quick-look' metric of weather conditions.

The classification shows the familiar diurnal pattern of dust devil activity. The persistence of dust devil images has been examined, and the presence of dust devils can be well-represented by a simple twostate Markov model.

At this site, at least, with a view of a few kilometers across, the image cadence selected $(1 / \mathrm{min})$ was effective for cataloging overall formation and migration rates of dust devils. Although the life-cycles of larger devils were reasonably described (about half of the larger devils were advected out of the field of view before decaying) a higher cadence would have been useful for more fully assessing the evolution of smaller, short-lived devils.

\section{Acknowledgments}

This work was funded by NASA through the Mars Fundamental Research Program grant number NNX12AI04G. Jani Radebaugh and Joe Spitale are thanked for assistance in the field. We acknowledge the Bureau of Land Management (NV-052, $9^{\text {th }}$ September 2015) for assessment that this research activity was in compliance with applicable land use regulations. Context meteorological data was acquired via http://mesowest.utah.edu/ . Two reviewers provided useful comments on the manuscript. 
Appendix

The Markov Model described in the text is implemented in the following ' $R$ ' Code which will reproduce figure 13.

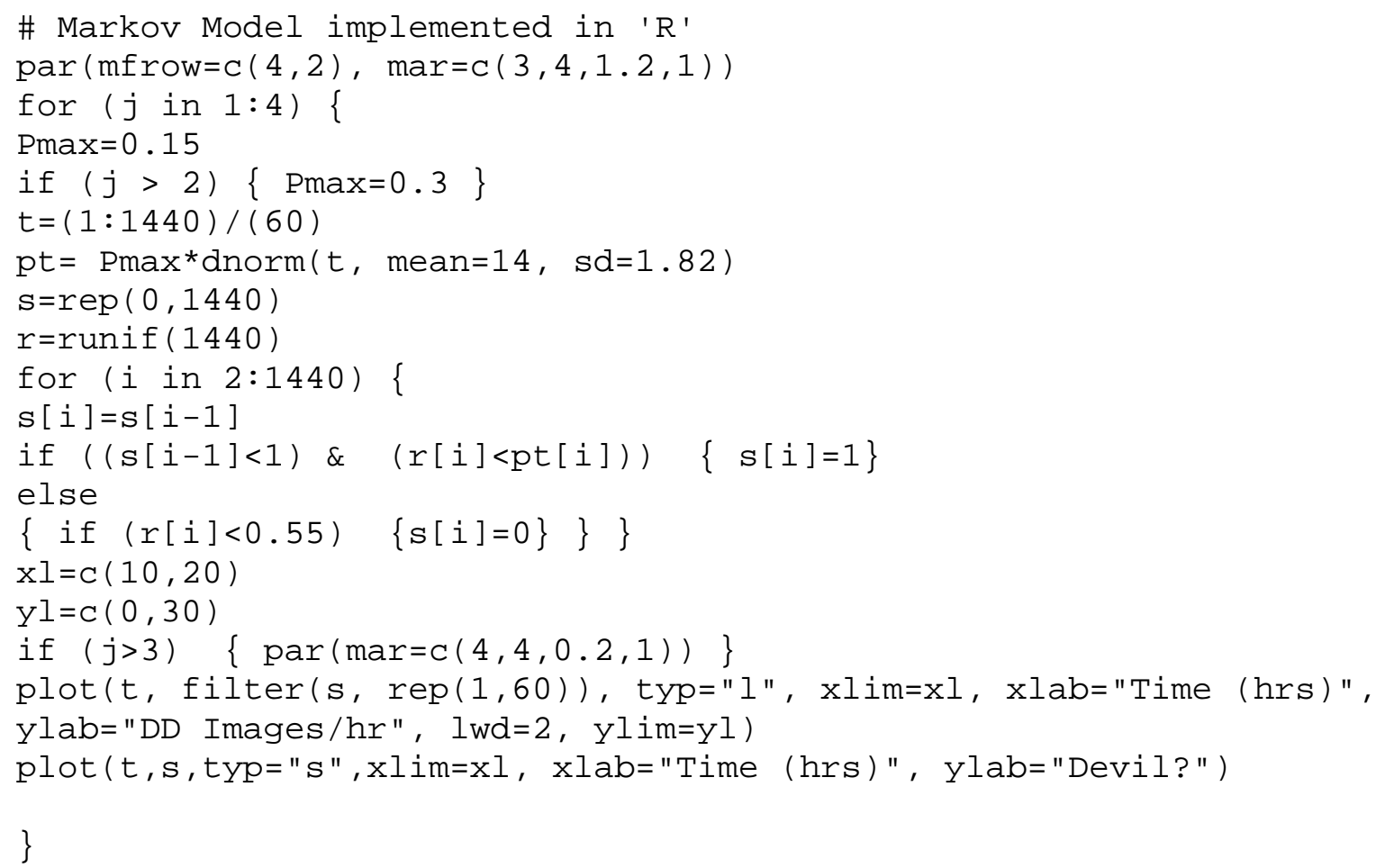


References

Balme, M. and R. Greeley, 2006. Dust Devils on Earth and Mars, Reviews of Geophysics, 44, RG3003

Balme, M.R., A. Pathare, S. M. Metzger, M. C. Towner, S. R. Lewis, A. Spiga, L. Fenton, N. O. Rennò, H. M. Elliott, F. A. Saca, T. I. Michaels, P. Russell and J. Verdasca, 2012. Field measurements of horizontal forward motion velocities of terrestrial dust devils: towards a proxy for ambient winds on Mars and Earth. Icarus 221, 632-645

Castano, A., Fukunaga, A., Biesiadecki, J., Neakrase, L., Whelley, P., Greeley, R., Lemmon, M., Castano, R. and Chien, S., 2008. Automatic detection of dust devils and clouds on Mars. Machine Vision and Applications, 19(5), 467-482.

Gillette, D. A. and P. C. Sinclair, 1990. Estimation of Suspension of Alkaline Material by Dust Devils in the United States, Atmospheric Environment, 24A, 1135-1142

Greeley, R., Whelley, P.L., Arvidson, R.E., Cabrol, N.A., Foley, D.J., Franklin, B.J., Geissler, P.G., Golombek, M.P., Kuzmin, R.O., Landis, G.A. and Lemmon, M.T., 2006. Active dust devils in Gusev crater, Mars: observations from the Mars exploration rover spirit. Journal of Geophysical Research: Planets, 111(E12). Doi: 10.1029/2006JE002743

Lorenz, R. D., 2013. The Longevity and Aspect Ratio of Dust Devils : Effects on Detection Efficiencies and Comparison of Landed and Orbital Imaging at Mars, Icarus, 226, 964-970

Lorenz, R. D. and A. Valdez 2011. Variable Wind Ripple Migration at Great Sand Dunes National Park, Observed by Timelapse Imagery, Geomorphology, 133, 1-10

Lorenz, R. D. and P. D. Lanagan, 2014. A Barometric Survey of Dust Devil Vortices on a Desert Playa. Boundary Layer Meteorology, 53, 555-568.

Lorenz, R. D. and B. K. Jackson, 2015. Dust Devils and Dustless Vortices on a Desert Playa Observed with Surface Pressure and Solar Flux Logging, GeoResJ, 5, 1-11 
Lorenz, R. D., B. Jackson and J. Barnes, Inexpensive Timelapse Digital Cameras for Studying Transient Meteorological Phenomena : Dust Devils and Playa Flooding, Journal of Atmospheric and Oceanic Technology, 27, 246-256, 2010

Lorenz, R.D., Norris, J.M., Jackson, B.K., Norris, R.D., Chadbourne, J.W. and Ray, J., 2014. Trail formation by ice-shoved" sailing stones" observed at Racetrack Playa, Death Valley National Park. Earth Surface Dynamics Discussions, 2, 1005-1022.

Lorenz, R. D., M. R. Balme, Z. Gu,, H. Kahanpää, M. Klose, M. Kurgansky, M. R. Patel, D. Reiss, A. P. Rossi, A. Spiga,T. Takemi, W. Wei. 2016. History and Applications of Dust Devil Studies, Space Science Reviews, 203, 5-37, doi:10.1007/s11214-016-0239-2

Mason, J.P., Patel, M.R., Lewis, S.R., 2014. The retrieval of optical properties from terrestrial dust devil vortices, Icarus $231,385-393$

Metzger, S.M., Balme, M.R., Towner, M.C., Bos, B.J., Ringrose, T.J., Patel, M.R., 2011. In situ measurements of particle load and transport in dust devils. Icarus 214, 766-772.

Pathare, A. V., M. R. Balme, S. M. Metzger, A. Spiga, M. C. Towner, N. O. Renno and F. Saca, 2010. Assessing the power law hypothesis for the size-frequency distribution of terrestrial and Martian dust devils, Icarus, 209, 851-852

Rafkin, S., B. Jemmett-Smith, L. Fenton, J. Ito, R. Lorenz, D. Tyler, 2016. Dust Devil Formation, Space Science Reviews, 203, 187-207,

Reiss, D., Fenton, L., Neakrase, L., Zimmerman, M., Statella, T., Whelley, P., Rossi, A.P. and Balme, M., 2016. Dust devil tracks. Space Science Reviews, 203(1-4), pp.143-181.

Sinclair, P.C., 1969. General characteristics of dust devils. Journal of Applied Meteorology, 8(1), pp.3245.

Snow, J.T. and McClelland, T.M., 1990. Dust devils at white sands missile range, New Mexico: 1. Temporal and spatial distributions. Journal of Geophysical Research: Atmospheres, 95(D9), pp.1370713721. 